\title{
Dihedral Cryptographic Technique
}

\author{
Zainab Fahad Mhawes \\ Al-Qadisiya university-College of education-Department of Mathematics \\ zainab.alnaseri@qu.edu.iq \\ Recived : 161012017 \\ Revised : 30\10\2017 \\ Accepted : 61212017
}

Available online : $\quad 21 / 1 / 2018$

DOI: $10.29304 / j q c m .2018 .10 .1 .337$

\begin{abstract}
:
This paper focuses on a new technique of cryptography in abstract algebra. We first give the necessary review on Dihedral group and cryptography .We definition a new alphabetic of characters by additive character "blank", thus we have (27) characters $\{26$ letters and "blank" $\}$, therefore we use modular 27 instead 26 such that we use the reflection and rotation which exists in Dihedral group to change the arrange of vectors of characters in plain text.
\end{abstract}

Keywords : Dihedral group, Cryptography ,Caesar Cipher ,Encryption Processes, Decryption processes.

\section{Mathematics Subject Classification: 68P25}

Introduction: Cryptography is one of the most important applications of algebra and number theory where the process is to change important information to another unclear one . The main goal of cryptography is to keep the integrity and security of this information there are many types of Cryptography techniques and we will try to consider some of them in this paper .
This paper consists of three paragraphs, where one includes some necessary definitions on dihedral groups. In second, we defined some necessary definitions on Cryptography. Third includes a suggested technique and some example and analysis of this technique. The programs of this paper write by using V.B. language . 


\section{Preliminary definitions in algebra:}

\subsection{Definition [4]:}

We say that $*: \mathrm{S} \times \mathrm{S} \rightarrow \mathrm{S}$, which defined by $(\mathrm{x}, \mathrm{y}) \rightarrow \mathrm{x}$ is a binary opration on a nonempty set $S$ if it is map .

\subsection{Definition[5]:}

A group $\left(\mathrm{G},{ }^{*}\right)$ is a nonempty set $\mathrm{G}$ with a binary operation * such that the following conditions are hold:

(i) $\left(x^{*} y\right)^{*} z=x^{*}\left(y^{*} z\right)$, for all $x, y, z \in G$

(ii) There exists an element e such that:

$$
\mathrm{x} * \mathrm{e}=\mathrm{x}=\mathrm{e}^{*} \mathrm{x} \text {, for all } \mathrm{x} \in \mathrm{G},
$$

(iii) For all $\mathrm{x} \in \mathrm{G}$ there is an element $\mathrm{x}^{-1}$ in $\mathrm{G}$ such that:

$$
\mathrm{x}^{-1} * \mathrm{x}=\mathrm{e}=\mathrm{x} * \mathrm{x}^{-1} .
$$

\subsection{Definition[4]:}

The set $D_{n}=\left\{r^{0}, r, r^{2}, \ldots, r^{n-1}, s, s r, s r^{2}, \ldots\right.$, $\left.\mathrm{sr}^{\mathrm{n}-1}\right\}$ is called the dihedral group and has order $2 \mathrm{n}$ with property $\quad \mathrm{sr}=\mathrm{r}^{-1} \mathrm{~s}$.

\subsection{Remarks[4]:}

$\mathrm{r}^{\mathrm{n}}=\mathrm{r}^{0}, \mathrm{~s}^{2}=\mathrm{r}^{0}$

\section{Preliminary definitions in Cryptography:}

\subsection{Definition[3]:}

Encryption is the process of changing the text of the content (data) to the symbols and Numbers are difficult to understand using the many and varied mathematical algorithms .

\subsection{Goals of Cryptography [1]:}

- Data privacy (confidentiality ).

- Data Authenticity (it came from where it claim).

- Data integrity(it has not been modified on the way) in the digital world.

\subsection{The fundamental objects of}

\section{Cryptography [2]:}

- Plaint text is the original data.

- Cipher text is the message changed by using some algorithms .

- Encryption is the processes which are changing the plaintext to cipher text.

- Decryption is the processes which are changing the cipher text to plaintext.

\subsection{Definition [4]:}

Let $\quad \mathrm{A}=\left[\begin{array}{c}a_{1} \\ a_{2} \\ \vdots \\ a_{n-1} \\ a_{n}\end{array}\right]$ be a vector then the cryptography transpose (CT) of A is $A^{C T}=\left[\begin{array}{c}a_{n} \\ a_{n-1} \\ \vdots \\ a_{2} \\ a_{1}\end{array}\right]$

We will introduce a new term in the following definition, that is the transpose of element. 


\subsection{Definition:}

Let $\quad \mathrm{A}=\left[\begin{array}{c}a_{0} \\ a_{1} \\ \vdots \\ \cdot \\ a_{n-1}\end{array}\right]$ be a vector and $\mathrm{a}_{\mathrm{k}}$ be an element of $\mathrm{A}$, then the transport of $a_{k}$ is $\left(a_{k}\right)^{T}=a_{(n-1)}-k$

We will define a new operation in the following definition, which it very import in our paper.

\subsection{Definition:}

Let $D_{n}=\left\{\mathbf{r}^{0}, \mathbf{r}, \ldots \ldots, \mathbf{r}^{\mathbf{n}-1}, \mathbf{s}, \mathbf{s r}, \ldots \ldots, \mathbf{s} \mathbf{r}^{\mathbf{n}-1}\right\}$ be a dihedral group then we can define the new operation :

$$
\begin{aligned}
& r^{k} a=\mathrm{a}+\mathrm{k} \bmod 27 \\
& r^{-k} a=\mathrm{a}-\mathrm{k} \bmod 27 \\
& s a=\mathrm{a}^{\mathrm{T}} \bmod 27 \\
& s r^{k} a=(\mathrm{a}+\mathrm{k})^{\mathrm{T}} \bmod 27
\end{aligned}
$$

\section{The suggested algorithm :}

Here we consider the blank is character, i.e the alphabet is 27 chars .

i- $\quad$ Encryption process :

1- Take positive integer number $\mathrm{n}$.

2- Construction Dihedral group $D_{n}$.

3- Cut block of plain text with length $2 \mathrm{n}$ character as :

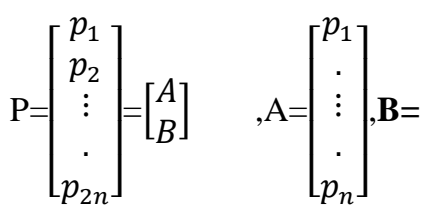

$\left[\begin{array}{c}p_{n+1} \\ \vdots \\ \cdot \\ p_{2 n}\end{array}\right]$

4- Apply the Dihedral operations (r,s):

$\mathrm{D}_{\mathrm{n}} \mathrm{p}=\left[\begin{array}{cc}\left(r^{k} a_{k+1}\right) & \bmod 27 \\ \left(s r^{k} b_{k+1}\right) & \bmod 27\end{array}\right], \mathrm{k}=0,1, \ldots, \mathrm{n}-1$

$\operatorname{DnP}=\left[\begin{array}{c}\left(\left[\begin{array}{c}0 \\ 1 \\ \vdots \\ n-2 \\ n-1\end{array}\right]+\left[\begin{array}{c}p_{1} \\ \vdots \\ \cdot \\ p_{n}\end{array}\right]\right) \bmod 27 \\ \left(\left[\begin{array}{c}0 \\ 1 \\ \vdots \\ n-2 \\ n-1\end{array}\right]+\left[\begin{array}{c}p_{n+1} \\ \vdots \\ \cdot \\ p_{2 n}\end{array}\right]\right)^{T} \quad \bmod 27\end{array}\right]$

For enhanced this technical we must encryption the first letter of plaintext because the first letter by using this technical stay the same letter always.

Encryption the first letter

$\mathrm{C}_{1}=\mathrm{p}_{1}+(2 * \mathrm{n}) \bmod 27$

\section{ii) Decryption process:}

$$
\begin{aligned}
& \mathrm{P}_{\mathrm{i}}=\mathrm{C}_{\mathrm{i}}-(2 * \mathrm{n}) \bmod 27 \\
& \mathrm{D}_{\mathrm{n}} \mathrm{C}=\left[\begin{array}{cc}
\left(r^{-k} a_{k+1}\right) & \bmod 27 \\
\left(r^{-k} s B_{k+1}^{T}\right) & \bmod 27
\end{array}\right]
\end{aligned}
$$

\subsection{Example:}

Take plain text="hello"

Encryption by using Dihedral Cryptographic Technique 


\section{Solution}

\section{1- Encrption}

Let $\mathrm{n}=2$

$\mathrm{D}_{\mathrm{n}}=\mathrm{D}_{2}=\left\{\mathrm{r}^{0}, \mathrm{r}, \mathrm{s}, \mathrm{sr}\right\},\left|\mathrm{D}_{2}\right|=4$

Hello $=\{$ Hell $\}+\left\{\mathrm{O}_{-}{ }_{-}\right\}$

"Hell" $\rightarrow \mathrm{P}_{1}=\left[\begin{array}{c}7 \\ 4 \\ 11 \\ 11\end{array}\right]=\left[\begin{array}{l}A \\ B\end{array}\right] \quad, \quad \mathrm{A}=\left[\begin{array}{l}7 \\ 4\end{array}\right]$

, $\mathrm{B}=\left[\begin{array}{l}11 \\ 11\end{array}\right]$

$\mathrm{D}_{1} \mathrm{P}_{1}=\left[\begin{array}{l}{\left[\begin{array}{l}0 \\ 1\end{array}\right]+\left[\begin{array}{l}7 \\ 4\end{array}\right]} \\ \left(\left[\begin{array}{l}0 \\ 1\end{array}\right]+\left[\begin{array}{l}11 \\ 11\end{array}\right]\right)_{T}\end{array}\right]=$

$\left.\left[\begin{array}{c}{\left[\begin{array}{l}7 \\ 5\end{array}\right]} \\ \left(\left[\begin{array}{l}11 \\ 12\end{array}\right]\right)_{T}\end{array}\right]=\left[\begin{array}{c}7 \\ 5\end{array}\right]\left[\begin{array}{c}7 \\ 15 \\ 14\end{array}\right]\right]=\left[\begin{array}{c}5 \\ 15 \\ 14\end{array}\right] \rightarrow \mathrm{HFPO}=\mathrm{C}_{1}$

The first letter:

$\mathrm{H} \rightarrow 7 \rightarrow 7+4=11 \rightarrow \mathrm{L}$

"O" $\rightarrow$ "O---“ $\left.\rightarrow \mathrm{P}_{2 \rightarrow} \rightarrow\left[\begin{array}{l}14 \\ 26 \\ 26 \\ 26\end{array}\right] \rightarrow\left[\begin{array}{l}14 \\ 26 \\ {[26} \\ 26\end{array}\right]\right]$

$\mathrm{D}_{2} \mathrm{P}_{2}=\left[\begin{array}{l}{\left[\begin{array}{l}0 \\ 1\end{array}\right]+\left[\begin{array}{l}14 \\ 26\end{array}\right]} \\ \left(\left[\begin{array}{l}0 \\ 1\end{array}\right]+\left[\begin{array}{l}26 \\ 26\end{array}\right)_{T}\right.\end{array}\right]=$

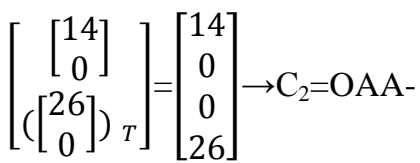

Then

$\mathrm{O} \rightarrow 14 \rightarrow 14+4=18 \rightarrow \mathrm{S}$

$\mathrm{P}=$ "Hello" $\rightarrow \mathrm{C}=$ "LFPOSAA-"

\section{2-Decryption :}

$\mathrm{C}=$ " LFPOSAA-“

$\mathrm{C}_{1}=$ "LFPO", $\mathrm{C}_{2}=$ "SAA-“

$\mathrm{C}_{1}$ :

$\mathrm{L} \rightarrow 11-4=7 \rightarrow \mathrm{H}$
$\left.\left.\mathrm{D}_{2} \mathrm{C}_{1}=\mathrm{D}_{\mathrm{n}}=\left[\begin{array}{c}7 \\ 5\end{array}\right]\right]\left[\begin{array}{c}15 \\ 14\end{array}\right]\right]=$

$\left[\begin{array}{l}-\left[\begin{array}{l}0 \\ 1\end{array}\right]+\left[\begin{array}{l}7 \\ 5\end{array}\right] \\ -\left[\begin{array}{l}0 \\ 1\end{array}\right]+\left[\begin{array}{l}15 \\ 14\end{array}\right]^{T}\end{array}\right]=$

$\left[\begin{array}{l}-\left[\begin{array}{l}0 \\ 1\end{array}\right]+\left[\begin{array}{l}7 \\ 5\end{array}\right] \\ -\left[\begin{array}{l}0 \\ 1\end{array}\right]+\left[\begin{array}{l}11 \\ 12\end{array}\right]\end{array}\right]=\left[\begin{array}{c}7 \\ 4 \\ 11 \\ 11\end{array}\right]$

$\rightarrow "$ HELL" $=\mathrm{P}_{1}$

$\mathrm{C}_{2}$ :

$\mathrm{S} \rightarrow 18-4=14 \rightarrow \mathrm{O}$

$\left.\mathrm{D}_{\mathrm{n}} \mathrm{C}_{2}=\mathrm{D}_{\mathrm{n}}=\left[\begin{array}{c}14 \\ 0 \\ 0 \\ 0 \\ 26\end{array}\right]\right]=$

$\left[\begin{array}{l}-\left[\begin{array}{l}0 \\ 1\end{array}\right]+\left[\begin{array}{c}14 \\ 0\end{array}\right] \\ -\left[\begin{array}{l}0 \\ 1\end{array}\right]+\left[\begin{array}{c}0 \\ 26\end{array}\right]^{T}\end{array}\right]=$

$\left[\begin{array}{l}-\left[\begin{array}{l}0 \\ 1\end{array}\right]+\left[\begin{array}{c}14 \\ 0\end{array}\right] \\ -\left[\begin{array}{l}0 \\ 1\end{array}\right]+\left[\begin{array}{c}26 \\ 0\end{array}\right]\end{array}\right]=\left[\begin{array}{c}14 \\ -1 \\ 26 \\ -1\end{array}\right]=\left[\begin{array}{c}14 \\ 26 \\ 26 \\ 26\end{array}\right]$

$\rightarrow$ "O---" $=\mathrm{P}_{2}$

Then $p=$ "Hello---"

\subsection{Example:}

Encryption the text:

College of education university of al Qadisiya

\section{Solution:}

$\mathrm{P}="$ College of education university of al Qadisiya"

$\mathrm{C}=$ " GPPOIHW

SGAVHVYZXJMMDVNRZFJHMU

C SGAZPAKZHJIRBBA" 


\subsection{Example:}

In example (3.1) and example (3.2) we take $\mathrm{n}=1$, now we will change value of $\mathrm{n}$ and compare between them:

$\mathrm{P}=$ ="Hello"

$\mathrm{n}=1 \rightarrow \mathrm{C}=$ " LFPOSAA-"

$\mathrm{n}=100 \rightarrow \mathrm{C}="$

SFNOSEFGHIJKLMNOPQRSTUVWXYZ ABCDEFGHIJKLMNOPQRSTUVWXYZ ABCDEFGHIJKLMNOPQRSTUVWXYZ ABCDEFGHIJKLMNOPQRA ZYXWVUTSRQPONMLKJIHGFEDCBA ZYXWVUTSRQPONMLKJIHGFEDCBA ZYXWVUTSRQPONMLKJIHGFEDCBA ZYXWVUTSRQPONMLKJ"

$\mathrm{n}=10 \rightarrow \mathrm{C}=$ " AFNOSEFGHIA ZYXWVUTS"

$\mathrm{P}=$ " College of education university of al Qadisiya"

$\mathrm{n}=1 \rightarrow \mathrm{C}=$ "GPPOIHW

SGAVHVYZXJMMDVNRZFJHMUC

SGAZPAKZHJIRBBA"

$\mathrm{n}=10 \rightarrow \mathrm{C}="$

WPNOILKGWOAVVDUVBLEETVPLZJXZ

QBC KSXVJUCRXJULBFFGHIA

ZYXWVUTS"

$\mathrm{n}=100 \rightarrow \mathrm{C}="$

NPNOILKGWOJPPGQPIZFFTOIESCQSJVA

DTLGIUJAMQWGYOSSTUVWXYZ

ABCDEFGHIJKLMNOPQRSTUVWXYZ

ABCDEFGHIJKLMNOPQRA

ZYXWVUTSRQPONMLKJIHGFEDCBA

ZYXWVUTSRQPONMLKJIHGFEDCBA

ZYXWVUTSRQPONMLKJIHGFEDCBA

ZYXWVUTSRQPONMLKJ"

We note that the complexity of cryptography increasing when the value $\mathrm{n}$ increasing.

\subsection{Frequency analysis:}

\begin{tabular}{|l||l||l|l|l|l||l|l||}
\hline $\mathrm{A}$ & $8.89 \%$ & $\mathrm{P}$ & $6.67 \%$ & $\mathrm{~F}$ & $2.22 \%$ & $\mathrm{~W}$ & $2.22 \%$ \\
\hline $\mathrm{H}$ & $8.89 \%$ & $\mathrm{~J}$ & $6.67 \%$ & $\mathrm{C}$ & $2.22 \%$ & $\mathrm{X}$ & $2.22 \%$ \\
\hline $\mathrm{Z}$ & $8.89 \%$ & $\mathrm{R}$ & $4.44 \%$ & $\mathrm{~K}$ & $2.22 \%$ & & \\
\hline \hline $\mathrm{V}$ & $6.67 \%$ & $\mathrm{~S}$ & $4.44 \%$ & $\mathrm{U}$ & $2.22 \%$ & & \\
\hline \hline $\mathrm{G}$ & $6.67 \%$ & $\mathrm{~B}$ & $4.44 \%$ & $\mathrm{D}$ & $2.22 \%$ & & \\
\hline $\mathrm{M}$ & $6.67 \%$ & $\mathrm{I}$ & $4.44 \%$ & $\mathrm{Y}$ & $2.22 \%$ & & \\
\hline \hline $\mathrm{O}$ & $2.22 \%$ & & & & & & \\
\hline \hline $\mathrm{N}$ & $2.22 \%$ & & & & & & \\
\hline
\end{tabular}

\section{FREQUENCY ANALYSIS}

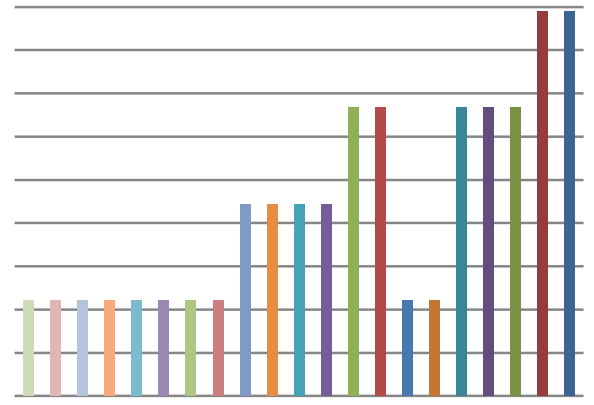

\section{Conclusions:}

1. The technique consists of an algebraic concept depends on recycling and displacement in forming the elements which led to the raising

Confidentiality and complexity level.

2. technical included some original ideas, whether in design or implementation making Her privacy.

3. encryption keys used random and difficult to detect. 


\section{References}

1- Alfred M. and paul v. ,A, Handbook of Applied Cryptography , CRC press ,2001.

2- Jonathan K. and Lindell Y., Introduction to modern Cryptography,CRC press, New york,2007.

3- Neal K. ,A course in number theory and Cryptography , spinger, 1994 .

$4-$

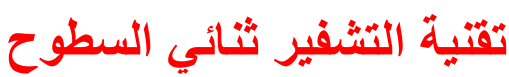

$$
\text { زينب فها مهوس }
$$

جامعة القادسية ـ كلية التربية ـ قسم الرياضيات

\section{zainab.alnaseri@qu.edu.iq}

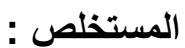

يركز هذا البحث على تقنية جديدة في التشفير في الجبر المجرد. في البداية اعطينا عرض ضروري عن زمر السطوح الثنائية و التشفير. عرفنا ابجدية جديدة للرموز بأضافة "الفراغ" ، لذلك اصبح لدينا (YV) رمز (Tr رمز و الفراغ ) ، لذلك استخدمنا معيار rV بدل rY حيث استخدمنا الانقلابات و التدوير الموجودة في زمرة السطوح الثنائية لتغيير ترتيب متجهات الرموز في لانص الصريح. 